\title{
Diagnostic Accuracy of a Limited Immuno-panel of Calretinin and Ber-EP4 for Diagnosis of Malignant Effusions
}

\author{
Nosheen Khurram, Tazeen Anis and Noshin Wasim Yusuf
}

\begin{abstract}
Objective: To differentiate between adenocarcinoma cells and reactive mesothelial cells (RMC) in serous effusions using a limited immuno-panel of Ber-EP4 and Calretinin.

Study Design: Descriptive study.

Place and Duration of Study: Department of Pathology, Allama Iqbal Medical College, Lahore, in collaboration with the Departments of Surgery, Pulmonology and Oncology, Jinnah Hospital, Lahore, from March 2015 to March 2016.

Methodology: Ninety-seven clinically and radiologically proven cases of peritoneal and pleural effusion and peritoneal wash of patients with suspicion of malignancy were included in the present study. Diagnostic accuracy of a limited immuno-panel of Calretinin and Ber-EP4 for diagnosis of malignant effusions was calculated using histopathology as gold standard.

Results: The sensitivity of Ber-EP4 for malignant cases was $98.6 \%$, specificity $100 \%$, positive predictive value (PPV) $100 \%$, negative predictive value (NPV) $96 \%$, and diagnostic accuracy $98.9 \%$. Sensitivity of Calretinin as positive staining for RMC was $79.2 \%$, specificity and positive predictive value (PPV) $100 \%$, negative predictive value (NPV) $93.6 \%$, and diagnostic accuracy $94.8 \%$.

Conclusion: Limited immuno-panel of Calretinin and Ber-EP4 had a high positive and negative predictive value and is cost-effective in resource limited set-up for identification of adenocarcinoma cells and reactive mesothelial cells in challenging cases of serous effusions.
\end{abstract}

Key Words: Reactive mesothelial cells, Adenocarcinoma cells, Serous effusion.

\section{INTRODUCTION}

Primary malignant tumors of the mesothelium are uncommon as compared to involvement of serous membranes by secondary metastatic tumor deposits. ${ }^{1}$ Among all malignant tumors, adenocarcinomas ${ }^{2}$ are the commonest which involve serous membranes with resultant malignant or reactive effusions. Cytological examination of aspirated body cavity fluids for diagnosis of malignant cells is a mandatory diagnostic procedure for correct tumor staging. ${ }^{3}$

Reactive mesothelial cells (RMC) are invariably present in effusions. 4 These cells can have variable cytological appearance and may resemble neoplastic cells phenotypically. ${ }^{5}$ In such cases, immunohistochemical markers are helpful in differentiating the reactive mesothelial versus malignant cells. ${ }^{6}$ Most studies suggest an extensive antibody panel comprised of a combination of mesothelial and epithelial markers.7,8 However, its application is not cost-effective. It remains non-feasible, therefore, for routine use in a resource limited setup. ${ }^{9}$

Department of Pathology, Allama Iqbal Medical College, Lahore, Pakistan

Correspondence: Dr. Nosheen Khurram, Department of Pathology, Allama Iqbal Medical College, Lahore, Pakistan E-mail: nosheenkhurram@gmail.com

Received: March 02, 2018; Accepted: September 07, 2018
In a developing country like Pakistan, there is a need to develop cost-effective diagnostic techniques. The present study was, therefore, designed to address this common problem of differentiating reactive and malignant exfoliated cells in clinically suspected malignant effusions by using a panel of two immunomarkers only.

The objective of this study was to evaluate the diagnostic accuracy of limited immuno-panel of two antibodies for discrimination of reactive mesothelial cells and malignant epithelial cells in effusions, Calretinin and BerEP4, respectively.

\section{METHODOLOGY}

Ninety-seven pleural and peritoneal effusion samples with provisional clinical diagnosis of benign effusion or suspected malignant effusion were collected from the outpatient and indoor departments of Surgery, Pulmonology and Oncology, Jinnah Hospital, Lahore.

The samples of pleural and peritoneal fluids and washings were received fresh in the Pathology Laboratory. The samples were examined for gross apperance, and findings were noted. Each sample was divided into two equal parts and transferred into two separate test tubes.

Test tube \#1 was processed for cytological examination. It was centrifuged at 2000 revolutions per minute for 5 minutes. The supernatant was discarded. Smears 
were prepared on glass slides from the deposit obtained after centrifugation. Minimum of two slides were prepared from each sample. One of these slides were air dried for Giemsa stain, 10 and other was fixed in ethanol for Hematoxylin and Eosin staining. ${ }^{11}$

Test tube \#2 was processed for cell block preparation. For hemorrhagic effusions 1 to 2 drops of $1 \%$ glacial acetic acid was added for lysis of RBC. The sample was centrifuged for 5 minutes at $1500 \mathrm{rpm}$. Supernatant was discarded. The deposit was then fixed in 1:1 solution of $10 \%$ formalin and centrifuged for 10 minutes again at $2500 \mathrm{rpm}$. The sediment was left in test tube for overnight. Then further sample processing and hematoxylin and eosin (H\&E) was done. ${ }^{12}$

The cell block slides were examined using the Olympus binocular microscope, CX-21. The scanner lens was used to examine the cellularity, architecture and pattern of the cells. Then low and high power objective lenses were used to examine the cytologic details to categorise the cell block as reactive, suspicious for positive malignant cells or positive for malignant cells. After that unstained slides were prepared from cell blocks and were subjected to immunohistochemistry (IHC) for confirmation of cell block diagnosis. For this purpose, two antibodies, Calretinin and Ber-EP4 were used. Calretinin is positive immunohistochemical marker for RMC and negative for adenocarcinoma cells while Ber-EP4 is positive marker for adenocarcinoma cells and is negative for RMC. ${ }^{13}$

Biopsy samples or surgically excised specimens of the suspected malignant cases were also received and were processed for histological examination. The cytological diagnosis of malignant cells was verified with the histopathological diagnosis on biopsy tissue and the immunohistochemistry results on cell blocks. For cases with provisional benign diagnosis clinic-radiological correlation and follow-up was used for verification. Study variables and information collected were entered into SPSS version 20.0 and analysed through its statistical programme. Immunohistochemical results were listed as positive or negative for presence or absence of adenocarcinoma cells and RMC. Descriptive statistics were presented as frequencies and percentages. Crosstabulation was done for IHC Ber-EP4 and IHC Calretinin with histopathology. Diagnostic accuracy was calculated using histopathology as gold standard.

\section{RESULTS}

Ninety-seven smears, prepared from centrifuged deposits of the aspirates, were examined for cytological features. Fifty-five $(56.7 \%)$ cases were reported positive for malignant cells; whereas, 21 (21.6\%) cases were reported as negative. Out of these negative 21 cases, three $(14.3 \%)$ cases showed acellular smears. In another $21(21.6 \%)$ cases, definitive diagnosis could not be rendered. These were reported as suspicious for malignant cells and clinical and radiological correlation was advised.

Cell blocks were prepared from the centrifuged deposit of all the 97 cases. Three unstained slides were made from cell block of each fluid, one slide was stained with $H \& E$ and rest of the 2 slides were used for application of Ber-EP4 and Calretinin each. Control of Ber-EP4 used was appendicular mucosa and control for Calretinin was malignant mesothelioma.

Out of 97 cases, Ber-EP4 showed positive staining (membranous staining) in $72(74.2 \%)$ cases and negative staining in $25(25.8 \%)$ cases. Clinical and radiological correlation was carried out. Histopathological diagnosis on biopsy tissue which was taken as gold standard revealed $73(75.3 \%)$ cases were actually to be positive for malignant cells; whereas, $24(24.7 \%)$ cases were benign. These results indicate that Ber-EP4 showed positivity in all the malignant effusion cases except one case. All the benign cases showed negative results.

Out of 97 cases, Calretinin showed positive (nuclear and cytoplasmic staining) staining in 19 (19.6\%) cases and negative staining in $78(80.4 \%)$ cases. All cases were clinically and radiologically correlated. This revealed 24 $(24.7 \%)$ cases were actually benign, and 73 (75.3\%) cases were malignant. Out of those 24 benign cases, only $19(79.2 \%)$ cases showed positive staining; whereas, all malignant effusions were negative for Calretinin staining. Results of Calretinin as positive staining in reactive mesothelial cells is also crosstabulated. Histological, clinical, and radiological correlation of all study cases were done.

Results of Ber-EP4 and Calretinin positivity and negativity were cross-tabulated with histopathological and clinical diagnosis. Sensitivity, specificity, positive predictive value (PPV), negative predictive value (NPV), and diagnostic accuracy were calculated.

Sensitivity of Ber-EP4 for malignant cases is $98.6 \%$, specificity is $100 \%$, PPV is $100 \%$ and NPV is $96 \%$ with diagnostic accuracy of $98.9 \%$.

Sensitivity of Calretinin as positive staining for RMC is $79.2 \%$, specificity is $100 \%$, PPV is $100 \%$, and NPV is $93.6 \%$ with diagnostic accuracy of $94.8 \%$ (Table I).

Table I: Diagnostic accuracy of IHC Ber-EP4 and IHC Calretinin.

\begin{tabular}{lcc}
\hline Diagnostic accuracy & IHC Ber-EP4 & IHC Calretinin \\
\hline Sensitivity & $98.6 \%$ & $79.2 \%$ \\
Specificity & $100.0 \%$ & $100.0 \%$ \\
PPV & $100.0 \%$ & $100.0 \%$ \\
NPV & $96.0 \%$ & $93.6 \%$ \\
Diagnostic accuracy & $98.9 \%$ & $94.8 \%$ \\
\hline
\end{tabular}

\section{DISCUSSION}

Distinction of reactive mesothelial cells from adenocarcinoma cells is critical in cytological diagnosis of body 
cavity effusions. The overlapping phenotypic features between these two types of cells pose a major diagnostic challenge in routine cytology practice. ${ }^{9}$

The work-up of body effusions includes examination of the cytological smears as a mandatory step; however, the diagnostic accuracy of serous effusion cytology using routine smear is low. ${ }^{14}$

Oyafuso et al. reviewed the cytological diagnosis of 4,297 serous fluid samples. According to their results, 1,982 false negative, 21 false positive, 1,588 true positive and 468 true negative were obtained; whereas, 161 were still suspicious for malignant cells and 77 were inconclusive. The authors inferred that cytological diagnosis alone cannot be $100 \%$ accurate for malignant effusions. ${ }^{15}$

The results of present study concur with these findings when out of 97 cases, 50 true positive, 5 false positive, 16 true negative, and 2 false negative results were obtained. This led to the diagnosis of $68 \%$ of the malignant cases and $66 \%$ of benign only on the basis of cytology. These results were confirmed with histopathological diagnosis and/or clinical and radiological diagnosis.

To complement the fluid cytology, technique of cell block enhances the sensitivity and specificity of cytological diagnosis. Nair, and Manjula, in their study on 148 effusion samples, compared the results of regular smears and cell blocks taking biopsy examination as the gold standard. Their results indicated that sensitivity of cell block was almost double than that of routine cytology. The results of present study tally with these authors, when $80 \%$ of malignant cases and $70 \%$ of benign/reactive cases were correctly diagnosed using the cell block technique. ${ }^{16}$

The cell block technique improves diagnosis by revealing better architectural pattern. ${ }^{17}$ Compact arrangement of cells in cell block along with least amount of background staining helps easy interpretation as compared to traditional smear. In addition, various sections can be obtained from single sample. Despite these advantages of cell block, in several cases due to variation in size and shape of reactive mesothelial cells and overlying phenotypic features with adenocarcinoma cells, ancillary techniques need to be used. Immunohistochemistry can greatly help in resolving challenging cases. ${ }^{18}$

A variety of mesothelial cell and epithelial cell antibodies have been used to assist in this differentiation. Studies have been performed to explore the diagnostic efficacy of different combinations of immunohistochemical markers for diagnosis of malignant serous effusions.

A study was done by Su et al., they also evaluated six immunohistochemical markers, three for metastatic adenocarcinoma cells (CEA, MOC31and Ber-EP4) and three for RMC (Calretinin, HBME1 and thrombomodulin). Sensitivity and specificity of each maker was calculated. Results showed $86.7 \%, 80 \%$, and $76.4 \%$ sensitivity for CEA, MOC31 and Ber-EP4, respectively and specificity calculated was $98.1 \%, 92.5 \%$ and $86.8 \%$, respectively. The sensitivity of Calretinin, HBME-1, and thrombomodulin for RMC was calculated as $83 \%$, $79.2 \%$, and $47.2 \%$, respectively. The specificity was $88.3 \%, 21.7 \%$, and $70 \%$, respectively. 19

Grefte and his co-authors studied six immunohistochemical markers. Among those were three mesothelial markers (Calretinin, EMA and HMGF1), and three epithelial markers (Ber-EP4, B72.3, and CEA). All six antibodies were applied on each cell block prepared from serous effusion samples. Their results showed that Calretinin is very sensitive marker for mesothelial cells. They also suggest that at least one antibody for epithelial cells along with Calretinin should be used for accurate diagnosis of malignant effusions. Finally, they concluded that Ber-EP4 is more sensitive as compared to rest of the two markers, by revealing $100 \%$ positive immunostaining in malignant cells and all reactive cases were negatively stained. 20 The present results also tally with the conclusion of these authors.

Politi et al. used HBME1, Calretinin, Moc 31, Ber-EP4 and BG 8 for differentiation of adenocarcinomas cells versus reactive mesothelial cells in 134 serous effusions. According to their findings, the sensitivity of HBME1 and Calretinin for mesothelial cells was $98 \%$ and $100 \%$, respectively. The sensitivity of the stains for adenocarcinoma cells was $86.25 \%$ for Moc $31,77.5 \%$ for Ber-EP4 and $67.5 \%$ for BG8; whereas, combined calculated sensitivity was $100 \%$. The results proved that Calretinin is an ideal marker for mesothelial cells. ${ }^{21}$

Fetsch and Abati calculated the percentage of immunoreactivity of frequently used antibodies for segregation of adenocarcinoma cells and reactive mesothelial cells in various studies. The findings showed $96 \%$ staining of adenocarcinoma cells with Ber-EP4 and 80 to $100 \%$ staining of reactive mesothelial cells with Calretinin. 22

These studies indicate that compared to other makers, Calretinin and Ber-EP4 has more sensitivity for mesothelial cells and adenocarcinoma cells, respectively. BerEP4 is considered to be one of the best available antibody for the panel used for differentiation of ACA and RMC in effusion cytology. However, its use in combination with a mesothelial marker is recommended for better diagnosis. ${ }^{23}$ As far as mesothelial markers are concerned, results indicated that Calretinin is specific and sensitive marker for reactive and neoplastic mesothelial cells. ${ }^{24,25}$

The number of antibodies that can be used in immunocytochemistry has increased dramatically over the past few years, and the future of diagnostic cytopathology will 
continue to expand as more and more immunohistochemical markers are validated and experimentally proved to be used. Yet for resource-limited set-ups, priority remains the cost-effectiveness; hence the hunt for a short but accurate immuno-panel.

Differentiation between RMC and $\mathrm{MM}$ is not possible with this limited immuno-panel of antibodies. Effusions containing cells of both malignant mesothelioma and reactive mesothelium, show positive immunoreactivity with Calretinin and negative results with Ber-EP4. Primary cause of malignant effusion cannot be diagnosed with this limited immuno-panel. Effusions due to malignant melanoma, lymphoma and sarcoma, although rare, cannot be diagnosed. Exclusion is possible, however.

\section{CONCLUSION}

Application of limited panel of Ber-EP4 and Calretinin on cell block preparations of serous fluid is cost-effective and time-saving technique, which can be used as regular diagnostic procedure along with cytological smear preparations. It is especially useful at resourcelimited centres with heavy workload as in our publicsector health institutions. The use of this combination of antibodies at the primary diagnostic level can aid in rapid and accurate diagnosis in morphologically difficult cases ensuring cost-effectiveness.

\section{REFERENCES}

1. Cibas ES, Ducatman BS. Cytology E-Book: Diagnostic Principles and Clinical Correlates. Elsevier Health Sciences, 2013.

2. Naylor B. Pleural, peritoneal, and pericardial effusions. Incomprehensive Cytopathology (3rd ed.) 2008; p. 515-77.

3. Nam HS. Malignant pleural effusion: medical approaches for diagnosis and management. Tuberc Respir Dis 2014; 76: 211-7.

4. Shidham VB, Atkinson BF. Cytopathologic diagnosis of serous fluids e-book. Elsevier Health Sciences; 2007.

5. Santwani PM, Vachhani JH. Analysis of diagnostic value of cytological smear method versus cell blocks method in body fluid cytology: Study of 150 cases. Ethiop J Health Sci 2014; 24:125-30.

6. Lozano MD, Panizo A, Toledo GR, Sola JJ, Pardo-Mindán J. Immunocytochemistry in the differential diagnosis of serous effusions. Cancer Cytopathol 2001; 93:68-72.

7. Szczepulska-Wójcik E, Langfort R, Roszkowski-Sliz K. A comparative evaluation of immunohistochemical markers for the differential diagnosis between malignant mesothelioma, non-small cell carcinoma involving the pleura, and benign reactive mesothelial cell proliferation. Adv Respir Med 2007; 75:57-69.

8. Lyons-Boudreaux V, Mody DR, Zhai J, Coffey D. Cytologic malignancy versus benignancy: how useful are the "newer" markers in body fluid cytology? Arch Pathol Lab Med 2008; 132:23-8.
9. Arora R, Agarwal S, Mathur SR, Verma K, lyer VK, Aron M. Utility of a limited panel of calretinin and Ber-EP4 immunocytochemistry on cytospin preparation of serous effusions: A cost-effective measure in resource-limited settings. Cytojournal 2011; 8:14.

10. Barcia JJ. The Giemsa stain: Its history and applications. Int $J$ Surg Pathol 2007; 15:292-6.

11. Layton C, Bancroft JD. Bancroft's theory and practice of histological techniques, expert consult: Online and print, 7: Bancroft's theory and practice of histological techniques. Elsevier Health Sciences; 2013.

12. Bista P. Comparison of the diagnostic accuracy of cell block with cytology smear in serous effusions. J Pathol Nepal 2013; 3:482-6.

13. Farmilo AJ, Stead RH. Handbook, immunochemical staining methods. Boenisch T, editor. DAKO Corporation; 2001.

14. Shivakumarswamy U, Arakeri SU, Karigowdar MH, Yelikar BR. Diagnostic utility of the cell block method versus the conventional smear study in pleural fluid cytology. J Cytol 2012; $29: 11$.

15. Oyafuso MS, Longatto AF, Bortolan J, Rahal P, Bisi $H$, Lombardo V. Cytological diagnosis of serous effusions in a cancer hospital in Brazil. Pathologica 1996; 88:128-31.

16. Nair GG, Manjula AA. Comparative study of cell-blocks \& routine cytological smears of pleural \& peritoneal fluids in suspected cases of malignancy. Indian J Pathol Oncol 2015; 2:61-8.

17. Thapar M, Mishra RK, Sharma A, Goyal V, Goyal V. Critical analysis of cell block versus smear examination in effusions. J Cytol 2009; 26:60.

18. Esteban JM, Yokota S, Husain S, Battifora H. Immunocytochemical profile of benign and carcinomatous effusions: a practical approach to difficult diagnosis. Am J Clin Pathol 1990; 94:698-705.

19. Su XY, Li GD, Liu WP, Xie B, Jiang YH. Cytological differential diagnosis among adenocarcinoma, epithelial mesothelioma, and reactive mesothelial cells in serous effusions by immunocytochemistry. Diagn Cytopathol; 39:900-8.

20. Grefte JM, de Wilde PC, Salet-van de Pol MR, Tomassen M, Raaymakers-van Geloof WL, Bulten J. Improved identification of malignant cells in serous effusions using a small, robust panel of antibodies on paraffin-embedded cell suspensions. Acta Cytol 2008; 52:35-44.

21. Politi E, Kandaraki C, Apostolopoulou C, Kyritsi T, Koutselini H. Immunocytochemical panel for distinguishing between carcinoma and reactive mesothelial cells in body cavity fluids. Diagn Cytopathol 2005; 32:151-5.

22. Fetsch PA, Abati A. Immunocytochemistry in effusion cytology. Cancer Cytopathol 2001; 93:293-308.

23. Wang B, Li D, Ou X, Yi Q, Feng Y. Diagnostic accuracy of BerEP4 for metastatic adenocarcinoma in serous effusions: A meta-analysis. PloS One 2014; 9:e107741.

24. Wieczorek TJ, Krane JF. Diagnostic utility of calretinin immunohistochemistry in cytologic cell block preparations. Cancer Cytopathol 2000; 90:312-9.

25. Barberis MC, Faleri M, Veronese S, Casadio C, Viale G. Calretinin. Acta Cytol 1997; 41:1757-61. 